\title{
Towards Research to Build Effective HIV Programmes in Uganda
}

\author{
Francis Ogojo Okello ${ }^{1}$, Ndungi wa Mungai ${ }^{1, *}$, Venkat Pulla ${ }^{2}$, Phillip Bwititi ${ }^{3}$ \\ ${ }^{1}$ School of Humanities and Social Sciences, Charles Sturt University, NSW Australia \\ ${ }^{2}$ School of Social Sciences, University of the Sunshine Coast, QLD Australia \\ ${ }^{3}$ School of Biomedical Sciences, Faculty of Science, Charles Sturt University, NSW Australia \\ *Corresponding Author: nmunga@csu.edu.au
}

\begin{abstract}
Behavior change interventions have been targeted by the Uganda government, donors and other stakeholders in tackling the HIV/AIDS epidemic. This paper examines the impact of these interventions on the populations' risk behaviours in HIV transmission or acquisition. In addition, this paper also examines qualitative aspects of HIV program development and implementation in Uganda and suggests both qualitative and quantitative research designs that will allow monitoring progress of program implementation so that effective changes can be made to get best outcomes. Implications of this data would assist both policy and public advocacy undertaken by social work professionals.
\end{abstract}

Keywords HIV, Prevention, Intervention, Funding, Social Work

\section{Introduction}

In the last fifteen years, the Ugandan government and its development partners and donors invested a lot of resources to improve the health of the population. Interventions targeting the human immunodeficiency virus (HIV), the infection responsible for the acquired immunodeficiency syndrome (AIDS), have been a central feature in the health interventions of Uganda. In this paper, HIV by itself is described from the context of the viral infection, while HIV and AIDS are described from the context of the psychosocial problems associated with HIV infection and its progression to the terminal stage of immunodeficiency.

With the establishment of the Uganda Aids Commission (UAC) in 1992 to ensure harmonization in the national response to HIV 1, the commission embarked on the development of the Multi-sectorial Approach to Control of AIDS (MACA), as an overall framework for guiding policy

1 The Uganda Aids Commission was established by Statute of Parliament and operates under the office of the president. and program development for the national response to HIV (United Nations General Assembly Special Session Country Progress Report, 2010). The main responsibility of the UAC was to create a platform under which a sector-wide approach (SWAp) to improve coordination, efficiency and equity in the health sector, which later culminated in the development of the Health Sector Strategic Plan (HSSP), 2000/2001 to 2004/2005 (Tashobya, Ssengooba and Cruz, 2006). According to Tashobya et al. (2006), the SWAp, by policy of the government, required donors to fund the implementation of a coordinated sector-wide strategy rather than standalone interventions. The strategies of the UAC urge for the fast tracking of implementation of universal access to HIV prevention, as has been the case with care and treatment for infected individuals (UAC, 2006; UAC; 2008).

Considering the trends in donor compared to government funding for the health sector, it is apparent that the health initiatives of the government of Uganda would be unsustainable without donor funding. Available data shows that donor funding to the health sector has increased at a pace much higher than the government funding. Between 2004 and 2006, the donor funded public sector health programs doubled from Uganda Shillings 254.85 billion to 507.26 billion2, compared with government-funded public sector health expenditure, which only increased by 10 Billion Uganda Shillings (4.5\%) from 219.56 to 229.88 billion in the same period (Örtendahl, 2007). Programs in HIV and AIDS, and prevention and treatment have received significant attention from donors. While momentous progress has been realized, a key question is whether the observed results are significant enough for the funding committed. In 2003/2004 to $2006 / 2007$, $\$ 234,348,403$ was spent on Uganda's national HIV and AIDS response, $94 \%$ of which came from donors, and $6 \%$ from the government (Ssengooba, Ekirapa, Kiwanuka and Maine, 2006). In 2006/2007, \$78,862,351 was spent on HIV prevention interventions in Uganda (Wabwire-Mangen, Odiit, Kirungi, Kisitu, Wanyama, 2009).

Although the early outcomes of Uganda's response to

2 Approximate value in US Dollar: \$139.8 million in 2004 to $\$ 278.25$ million

ISSN: 2332-6840 (Online) 2332-6832 (Print) Copyright (C) 2014 Horizon Research Publishing 
mitigate HIV transmission have been dramatic, recent results unfortunately show that the HIV prevalence that was declining has stagnated in some sites, and an increasing prevalence is being observed in some locations. The Ministry of Health (2009) HIV epidemiological results in Uganda show that by 1992, the urban HIV prevalence had peaked at $30 \%$, while in rural areas it was $10 \%$. Wabwire-Mangen et al, (2009) showed that from 1989 to1992, HIV prevalence in Uganda, which had peaked at $18 \%$, had declined to a stable average of $6.3 \%$ between 2002 and 2005. These observations are of concern to all stakeholders involved in the fight against HIV and AIDS, particularly because the increase is happening at a time when the response to HIV has increased in funding and programs. Shafer et al. (2008) in their study on HIV prevalence and incidence in the rural population of south western Uganda found that HIV prevalence, which had declined from $8.5 \%$ in $1990 / 1991$ to $6.2 \%$ in $1999 / 2000$, had increased to $7.7 \%$ in 2004/2005. The same study found that incidence per 1000 person-years at risk dropped from 7.5 in 1990 to 4.1 in 1998, and thereafter increased to 5.0 by 2004 . The study by Wabwire-Mangen et al. (2009) reports that an estimated 91,546 new infections occurred in Uganda in 2008 among the 15-49 year olds, and the main modes of transmission were: mutual monogamous heterosexual (43\%), multiple partnerships (24\%) and the partners of individuals reporting multiple partnerships (22\%). One of the advocated preventative measures to combat transmission is faithfulness to one sexual partner, but the effectiveness of this strategy comes to question given the evidence that transmission was highest in this group, posing the need to further investigate the effect of behaviour change communication messages in changing HIV transmission or acquisition behaviours.

The earliest reported cases of HIV in Uganda were identified in the early 1980s in Rakai District (Monico, Tanga and Nuwagaba, 2001). Since then, interventions to combat HIV intensified and biomedical and social interventions have increased with the formation of bodies such as the National Aids Control Program (NACP), the Uganda AIDS Commission, the Aids Information Center and various facility-based medical interventions established as the response to the epidemic intensified. Various social responses through the formation of nongovernmental organizations (NGO) working in HIV prevention and support for infected and affected individuals were also been established, with the Aids Support Organization (TASO) being a key pioneer. It is evident from the decline in HIV prevalence in the 1990's that the interventions implemented then were effective in combating HIV. What is therefore puzzling is the rising prevalence of HIV that has followed the decline. From the onset, Uganda's HIV response received a lot of publicity, which attracted international attention and funding. Programs to fight against HIV increased in the late 1990s to-date, and high levels of population awareness about the modes of transmission and prevention have been observed in various demographic health surveys 3 . The reversal of that downward trend therefore raises questions requiring empirical investigation as there has been no satisfactory explanation to date.

It is important for new research to investigate and increase understanding of:

- the elements of the early programs that resulted in a decline in HIV

- changes that may have occurred in the HIV programs in the later years of the epidemic

- whether the successful interventions that were implemented then had become outdated

- whether the increases in HIV awareness really contributed to increase in adoption of preventive behaviors, and if so, what changed the awareness

- if those preventive behaviors had an impact on HIV prevalence

- the main factors for the recent stagnation or gradual increase in prevalence

- if there is a need to develop new approaches to fight HIV considering the new knowledge about HIV we now have, increased funding and advancements in biomedical field related to HIV

These and other questions call for scientific inquiry to generate evidence for HIV programs to consider in decision-making and design and implementation of new programs. It is paramount to determine if new approaches are needed to combat HIV, especially taking into consideration the fact that funding for the health sector has increased significantly, health systems have improved and biomedical interventions in HIV prevention and treatment have advanced; these are some elements that are key in the fight against HIV.

Although the Ugandan government and donors have made significant investments to fight HIV and AIDS, and have achieved a number of results, the exact impacts of these interventions have not been adequately evaluated. This gap in knowledge may have contributed to the observed increases in HIV prevalence due to a failure to generate new information for effective prevention of new infections. Empirical studies to determine whether the changes in behavior related to HIV control are correlated with the changes in funding commitments have not been undertaken. Such information would allow for the determination of the effectiveness of existing HIV control/preventative programs. It is important to be mindful of the fact that most countries including some donor countries are going through recession. Therefore operating in a tight fiscal environment calls for efficiency in resource utilization, with emphasis placed on directing funds to interventions that are likely to result in higher impacts.

\section{Social Work and HIV and AIDS}

From behavioural interventions that are within the ambit 
of the social work profession, the prevalence of depression is common among people living with HIV and AIDS, and contributes to a wide range of worsened HIV-related outcomes, including AIDS-related mortality. Targeting modifiable causes of depression, either through primary or secondary prevention, may reduce suffering as well as improve HIV-related outcomes (Tsai et al., 2012). Yet another area of social work intervention such as lack of disposable incomes and insecurity related to food and employment are highly conspicuous in the sector. Tsai et al., (2012) analysed data from a cohort of 456 persons from the Uganda AIDS Rural Treatment Outcomes study-an ongoing prospective cohort of persons living with HIV/AIDS (PLWHA) initiating HIV antiretroviral therapy in Mbarara, Uganda-and found that Social support, HIV disclosure, and internalized HIV-related stigma were associated with food insecurity; these associations persisted after adjusting for household wealth, employment status, and other previously identified correlates of food insecurity. The adverse effects of internalized stigma persisted in a lagged specification, and the beneficial effect of social support further persisted after the inclusion of fixed effects.

HIV and AIDS have also caused a number of children to be orphaned. An overwhelming proportion of child infections and orphaned children are in sub-Sahara Africa (Foster and Williamson, 2000). A compelling reason to focus on the AIDS problem is that with the high impact on children, the problem will continue to affect the community even if the cure for HIV was found. Studies elsewhere in the world examined the relationships between several risk and protective factors regarding depression among AIDS orphans and provided important information to guide the development of mental health interventions for AIDS orphans. Previous research demonstrated that perceived social support, social work interventions, development of trusting relationships and future orientation offer multi-level protection for the mental health of orphans (Wang, Li, Barnett, Zhao, Zhao, and Stanton, 2012)

1) Gender is another important issue in the entire area of HIV and AIDS. The conception of a woman as victim of pain and disease resonates in all African Societies (Zhou and Landa, 2013). Evidence from semi-autobiographical/life narratives by women authors who have experienced the harrowing pain and consequent trauma of HIV and AIDS indicates that successful resilience and coping begins with woman. Further discriminated and predominantly exposed, the women are 'involved in a host of hardships, namely: poverty, stigma and discrimination as well as emotional and spiritual turmoil' (Zhou and Landa, 2013, p 399). While the Ugandan society collectively copes with HIV directly or indirectly, the burden is greatly bone by the women, particularly those who are infected. They do not only have to deal with the infection, but are also saddled with several family-related stresses such as childcare, food and household chores. Gender based violence and unemployment are common in HIV and AIDS infected individuals. Furthermore, some cultural practices and beliefs have also worked in unison to corner women into further subjugation. The authors believe that addressing issues of culture will be paramount in relation to building the coping abilities of women and in increasing their ability to handle difficulties more positively. The stigma of HIV also intensifies the poverty, stress, and insecurity endemic to many resource-limited settings (Tsai et al., 2011), resulting in worsened mental health, itself an important determinant of AIDS-related mortality. It is amply clear that gender-based social work interventions in HIV and AIDS area are equally required in Uganda and Africa in general. Once again this highlights the role of behavioral programs through planned social work interventions. Trusting relationships with caregivers provide the most immediate sources of psychosocial support. Until we can better understand how to effectively intervene to reduce the stigma of HIV, it will continue to adversely affect the well-being of HIV-infected persons and undermine both treatment and prevention efforts throughout sub-Saharan Africa.

2) While social workers have been central to the fight against HIV and AIDS to-date, the concern for this pandemic to social workers is still evident. HIV and AIDS present both a health and social problem that predominantly affects people who are already marginalised. Social workers are dedicated to work with the less privileged in society and have an ethical obligation to combat the discrimination and other injustices connected to AIDS (Jaoko, 2012). Social workers are also called upon to inform themselves of the range of issues involved in HIV/AIDS in order to be effective in direct intervention, research and policy (Vincent, 2000). Social workers roles include prevention, social advocacy, policy development and counseling. Africa is the region most affected by HIV/AIDS even though the epidemic is a global one.

In Uganda, coupled with the strong political will, some of the earliest successes in the HIV response can be attributed to the activities of social workers. A strong emphasis of HIV programs was placed on socio-behavioural dimensions of HIV prevention. Social workers were, and continue to be at the forefront in combating HIV through social mobilization, support for people living with HIV (PLHIV) and those affected due to HIV infection of a family or community member. A study by Ankrah (1992) shows that the government, through the Social Rehabilitation Ministry, responded to the social welfare problems resulting from HIV - such as providing support to widows and orphans. The same study describes the role of social workers at the ministry of health in health education, social mobilization, 
research, counseling, program management and many other areas of support. These interventions happened well before the formation of various non-governmental organizations, religious-based interventions and other support groups. Considering the stigma associated with HIV during the early years of its discovery and the improvement in attitudes towards HIV infected individuals and knowledge about HIV, it is undisputable that social workers were central to the early successes in HIV prevention and support in Uganda.

\section{Statement of the Problem}

Over the years, the governments of Uganda and donor organizations have committed substantial funding to implement health programs in Uganda to ameliorate the health of the population. Projects targeting prevention and treatment of HIV and AIDS have received substantial funding and support. The government and donors have specified the health outcomes they expect from these investments. However, studies that empirically evaluate the trends on target indicators have not been conducted.

In the case of HIV programs, donor funding has mainly targeted at interventions that promote adoption of HIV prevention behaviours, increase uptake of voluntary counseling and testing, delivery of treatment and care for people living with HIV and provision of social support interventions for individuals and families infected or affected by HIV (Global fund, Country Proposal for Round 9, 2009). Non-empirical data shows that prevalence of HIV declined substantially in the late 80 's and early 90 's. However, new data suggests that HIV prevalence is stagnating while some other studies show an increasing prevalence in some sites (Shafer et al., 2008; Wabwire-Mangen et al, 2009, Ministry of Health Epidemiological Surveillance Report, 2009).

While it would be expected that the significant investment to promoting HIV prevention would lead to a continuous drop in prevalence as had been the case historically, descriptive data suggests otherwise. As understanding of the extent to which the target populations have been exposed to interventions, and determining the effectiveness of those interventions in changing behaviors is important to future program decisions.

The Uganda Demographic Health Survey conducted by Uganda Bureau of Statistics and Macro International Inc. (2011), shows that awareness of HIV is almost universal, and knowledge of HIV prevention methods is high in the Ugandan population. Regardless, $19.4 \%$ of the men had two or more sex partners in the past 12 months, and among those, only $18.3 \%$ used condoms at the last sex encounter. United Nations General Assembly Special Session (UNGASS) Country Progress Report of the Uganda Aids Commission (2010) shows that behavioral indicators for HIV prevention have worsened, particularly an increase in multiple concurrent partnerships. The same report states that the epidemic has shifted from people in single casual relationships to those in long-term stable relationships; $43 \%$ of new HIV infections are among monogamous relationships and $46 \%$ are among persons reporting multiple partnerships. Such data raises questions about what has happened to the hitherto successful HIV prevention in Uganda. Empirical evidence of the effectiveness of behaviour change campaigns to reduce risky sexual behaviours has not been conducted, yet such investigation is necessary to establish if the investment in behaviour change interventions is effective in reducing practices that pre-dispose people to HIV infection and transmission

Considering the large investment to influence the population to adopt HIV prevention behaviors (such as condom use, changes to cultural norms of wife inheritance, safe disposal of sharp medical object), a key research question that remains unanswered is whether there is a correlation between exposure to prevention information and adoption of the expected health behavior, and whether the adoption of the prevention behavior is correlated with the desired health outcome and impact. Understanding these correlations will elucidate the effectiveness of current interventions in combating HIV, and if necessary influence the redesign of interventions and promotion of efficient use of scarce resources to support effective interventions.

\section{Moving Forward with Research}

While it is important to determine if the trends in the outcomes of government and donor interventions in HIV programs have been effective at the population level and whether the changes in the trends in selected indicators are significant, it is equally important to consider interventions and research outcomes from a social work perspective. Future studies could:

- Determine the most effective approaches to stigma reduction

- Help identify and promote adoption of synergistic strategies that target both the stigmatized through employment and social security provision related interventions

- Help figure out ways for dealing with stigmatizing factors through educational or social work generated interventions

- Define interventions related to building coping and making communities resilient (Pulla, 2013; Zhou and Landa, 2013)

Areas that future research needs to address from policy and program perspective need to include:

- Determining if the changes in the trends of key health indicators in HIV interventions supported by government and donors are significant

- Measuring the effectiveness of exposure to HIV behavior change communication initiatives in reducing risk behaviours and morbidity

- Determining factors that influence successful implementation of HIV programs

- Generating data that will support future strategies for improving implementation of HIV programs.

Such research is of importance for a number of reasons; 
firstly, the data will assist the government, donors and development partners to evaluate programs in HIV and secondly, the information will generate new knowledge on lessons to justify improvements in the delivery of health programs. Previous results of projects in HIV programs have mostly been descriptive, and for this reason have not clearly demonstrated the impact in relation to resource inputs.

While presenting a list of key indicators of future research needs, the authors also present two research designs that may be applicable: the quantitative and the qualitative. The quantitative design could use inferential statistics to test significance of observed trends in the indicators measured. Additionally, it assists to review statistics on service delivery data on HIV testing, treatment and prevention of mother to child transmission programs to establish trends in uptake of services. The qualitative design can utilize in-depth interviews and focus group discussions (FGDs) with key individuals involved in program implementation, ministry of health staff, representatives from donor agencies and with target beneficiaries. The analysis needs to examine if the trends are significant when confounding factors are controlled for. The range of data that would be collected from these approaches is shown on Table 1.

The qualitative design should use in-depth interviews with categories of key individuals such as: major international implementing agency of HIV programs; their perception of the results they achieved; hindrances to maximization of results achievement and; strategies for improved programs. Donor agencies that provide funding for HIV will also obtain information about past funding priorities and their perceptions of the results, challenges and strategies to overcome such challenges.

Additionally, FGDs will be conducted with target beneficiaries to investigate their perception of donor interventions, the results observed in their communities and how they feel donor programs can be better implemented for greater results achievement. The beneficiaries include sexually active men and women. These groups have been the major target for behavior change communication and promotion of medical services utilization for HIV. Social work has an important role to highlight the problems experienced by children in these circumstances and ensure that their voices are heard and their interests taken into consideration in program planning, policy making and in research agendas.

\section{Conclusions}

Upward trends in HIV prevalence have been noted in various countries, including developed countries (Presanis et al., 2010; McInnes et al., 2008; Calleja, 2002; Shafer et al., 2008). The dread associated with HIV in the early years of its discovery seems to have played a big role in shaping positive prevention behaviours due to the fear about the terminal nature of the HIV disease and the focus of programs on disseminating information about prevention behaviors. With the improvements in knowledge about HIV and advances in the biomedical fields of HIV management, it is possible to suspect complacency of the population as one of the main reasons for the stagnation of the decline in some countries and an upward surge in others. Researchers however need to investigate this and other assumptions to determine new strategies or improvements to current programs to fortify HIV prevention outcomes. Uganda provides an ideal setting for this study considering the evidence that shows early decline, stagnation and the recent upward trends in prevalence. The findings from this research will be relevant to HIV prevention programs in other countries experiencing similar reversals in their progress in combating HIV and AIDS. Social work as a profession in the forefront in addressing the problems arising from HIV and AIDS has a duty to conduct research to support the profession's role in advocacy and policy changes that help mitigate social problems resulting from illnesses such as HIV.

Table 1. Indicators for measuring trends in health outcomes

\begin{tabular}{|c|c|}
\hline No. & Outcomes that need measuring \\
\hline 1 & Comprehensive knowledge about HIV \\
\hline 2 & Attitudes towards people living with HIV \\
\hline 3 & Prevalence of HIV \\
\hline 4 & Prevalence of other sexually transmitted diseases \\
\hline 5 & Use of HIV prevention behaviors (condom use at last risky sex, number of multiple sex partners, abstinence, faithfulness) \\
\hline 6 & Proportion of youth initiating sex at a higher age \\
\hline 7 & HIV testing and receipt of results (knowledge of own HIV status) \\
\hline 8 & Uptake of HIV testing among antenatal clients \\
\hline 9 & $\begin{array}{c}\text { Adoption of positive behaviors for prevention of HIV acquisition and transmission among individuals who know their HIV test } \\
\text { result }\end{array}$ \\
\hline 10 & Uptake of care and support services among individuals who are HIV positive \\
\hline
\end{tabular}




\section{REFERENCES}

Ankrah E.M. (1992). Aids in Uganda: Initial Social Work Responses. Journal of Social Development in Africa. 7(2), 53-61.

Callejaa, J.M.G., Walker N., Cuchi P., Lazzari S., Ghysa P.D., \& Zacarias F. (2002). Status of the HIV/AIDS epidemic and methods to monitor it in the Latin America and Caribbean region. AIDS 16(suppl. 3): S3-S12.

Foster, G., \& Williamson, J. (2000). A review of the current literature of the impact of HIV/AIDS on children in sub-Saharan Africa. AIDS 2000, 14 (supp.3): s275-s284. http://storage.globalcitizen.net/data/topic/knowledge/uploads/2011 070615288705.pdf

Jaoko, J. (2012). HIV/AIDS issues in social work practice. Presented at NACSW Convention October 2012, St. Louis, MO. Retrieved from http://www.nacsw.org/Publications/Proceedings2012/JaokoJHIV.p df

McInnes, C.W., Druyts, E., Harvard, S.S., Gilbert, M., Tyndall, M.W., Lima, V.D., . . . \& Hogg R.S. (2008). HIV/AIDS in Vancouver, British Columbia: a growing epidemic. Harm Reduction Journal. 6(5). doi:10.1186/1477-7517-6-5

Ministry of Health. (2000). Health Sector Strategic Plan (HSSP), 2000/2001 to 2004/2005. Kampala. Ministry of Health.

Ministry of Health. (2009). Global Fund Country Proposal for Round 9. Kampala. Ministry of Health.

Ministry of Health. (2009). The HIV/AIDS epidemiological surveillance report. Kampala. Ministry of Health.

Monico, S.M., Tanga, E.O., \& Nuwagaba A. (2001). Uganda: HIV and AIDS-related Discrimination, Stigmatization and Denial. Geneva. UNAIDS.

Örtendahl, C. (2007). The Uganda health SWAp: new approaches for a more balanced aid architecture? London. HLSP Institute.

Presanis, A.M., Gill O.N., Chadborn, T.R., Hill, C., Hope, V., Logan, L., . . . \& De Angelis, D. (2010). Insights into the rise in HIV infections, 2001 to 2008: a Bayesian synthesis of prevalence evidence. AIDS. 24(18), 2849-2858

Pulla, V. (2013). Contours of Coping and Resilience, ( p.1-24) in Pulla, V., Shatte, A. \& Warren, S. (Eds). (2013). Perspectives on Coping and Resilience, New Delhi: Authorpress Global Network ISBN 978-81-7273-715-3

Shafer, L.A., Biraro, S., Nakiyingi-Miiro, J., Kamali, A., Ssematimba, D., Ouma, J., . . . \& Grosskurth, H. (2008). HIV prevalence and incidence are no longer falling in southwest Uganda: evidence from a rural population cohort 1989-2005.
AIDS, 22(13), 1641-1649). doi: 10.1097/QAD.0b013e32830a7502

Ssengooba, F., Ekirapa, E., Kiwanuka, S., \& Maine, S. (2006). Sector Based Assessment of HIV/AIDS Spending in Uganda. Kampala. Makerere University School of Public Health.

Tsai, A.C., Bangsberg, D.R., Emenyonu, N., Senkungu, J, K., \& Martin, J.N. (2011) The social context of food insecurity among persons living with HIV/AIDS in rural Uganda. Soc Sci Med 73: 1717-1724. doi: 10.1016/j.socscimed.2011.09.026

Tsai, A.C., Bangsberg, D.R., Frongillo, E.A., Hunt, P.W., Muzoora, C., Martin, J.N., \& Weiser,S,D, (2012). Food insecurity, depression and the modifying role of social support among people living with HIV/AIDS in rural Uganda. Social Science and Medicine. 74(12), 2012-2019. ISSN 0277-9536. Retrieved from http://www.sciencedirect.com/science/article/pii/S0277953612002 079

Tashobya, C.K., Ssengooba, F., \& Cruz, V.O. (Eds). (2006). Health Systems Reforms in Uganda: processes and outputs. London. Health Systems Development Programme, London School of Hygiene and Tropical Medicine

Uganda Bureau of Statistics and Macro International Inc. (2011). Uganda Demographic and Health Survey. Calverton, Maryland, USA. UBOS and Macro International Inc.

Uganda Aids Commission. (2006). Rapid assessment of trends and drivers of HIV epidemic and effectiveness of prevention interventions in Uganda. Kampala. UAC.

Uganda Aids Commission. (2008). National HIV and AIDS Strategic Plan 2007/08 - 2001/12. Moving towards universal access. Kampala. UAC.

Uganda Aids Commission. (2010). United Nations General Assembly Special Session. Kampala. UAC.

Vincent, L (ed.). (2000). HIV/AIDS at 2000: a source book for social workers. New York. Allyn and Bacon

Wabwire-Mangen F, Odiit M, Kirungi W, Kaweesa D.K, Okara J.W. (2009). Uganda HIV Prevention Response and Modes of Transmission Analysis. Kampala. Uganda National Aids Commission.

Wang, B., Li X, Barnett, D., Zhao, G., Zhao,. \& J, Stanton, B. (2012). Risk and protective factors for depression symptoms among children affected by HIV/AIDS in rural China: A structural equation modelling analysis. Social Science \& Medicine, 74(9), 1435-1443. Retrieved from

http://www.sciencedirect.com/science/article/pii/S0277953612001 128

Zhou, S \& Landa, N. (2013). Life Narratives Mirroring the feminization of HIV and Aids Trauma: Zimbabwean Perspectives of Coping and Resilience, ( p 399-318) in Pulla, V., Shatte, A. \& Warren, S. eds (2013). Perspectives on Coping and Resilience. New Delhi: Authorpress Global Network ISBN 978-81-7273-715-3. 